\title{
Electrocardiographic exercise testing in adults: performance and interpretation. An expert opinion of the Polish Cardiac Society Working Group on Cardiac Rehabilitation and Exercise Physiology
}

\author{
Krzysztof Smarż ${ }^{1}$, Tomasz Jaxa-Chamiec ${ }^{1}$, Tadeusz Bednarczyk², Bronisław Bednarz ${ }^{1}$, Zbigniew Eysymontt ${ }^{3}$, \\ Michał Gałaszek ${ }^{3}$, Anna Jegier ${ }^{4}$, Iwona Korzeniowska-Kubacka ${ }^{5}$, Artur Mamcarz ${ }^{6}$, Agnieszka Mawlichanów ${ }^{7}$, \\ Ryszard Piotrowicz ${ }^{5}$, Jerzy Rybicki ${ }^{8}$, Ewa Straburzyńska-Migaj ${ }^{9}$, Dominika Szalewska ${ }^{10}$, Jadwiga Wolszakiewicz \\ 'Department of Cardiology, Centre of Postgraduate Medical Education, Grochowski Hospital, Warsaw, Poland \\ ${ }^{2}$ Cardiac Rehabilitation Unit, Voivodship Clinical Hospital No. 2, Rzeszow, Poland \\ ${ }^{3}$ Silesian Rehabilitation and Prevention Centre, Ustron, Poland \\ ${ }^{4}$ Department of Sports Medicine, Medical University of Lodz, Lodz, Poland \\ ${ }^{5}$ Department of Cardiac Rehabilitation and Noninvasive Electrocardiology, Institute of Cardiology, Warsaw, Poland \\ ${ }^{6} 3^{\text {rd }}$ Department of Internal Medicine and Cardiology, $2^{\text {nd }}$ Faculty of Medicine, Medical University of Warsaw, \\ Miedzylesie Specialist Hospital, Warsaw, Poland \\ ${ }^{7}$ Cardiac Rehabilitation Unit, Krakow Specialist Hospital, Krakow, Poland \\ "SPZOZ "REPTY", Upper Silesian Rehabilitation Centre, Tarnowskie Gory, Poland \\ ${ }^{9} 1^{\text {st }}$ Chair and Department of Cardiology, Poznan University of Medical Sciences, University Hospital of Lord's Transfiguration, Poznan, Poland \\ ${ }^{10}$ Department of Rehabilitation, Medical University of Gdansk, Gdansk, Poland
}

\begin{abstract}
Electrocardiographic (ECG) exercise stress test has been a major diagnostic test in cardiology for several decades. Ongoing technological advances that have led to a wide use of imaging techniques and development of new guidelines have called for a revised and updated approach to the technique and interpretation of the ECG exercise testing. The present document outlines an expert opinion of the Polish Cardiac Society Working Group on Cardiac Rehabilitation and Exercise Physiology regarding the performance and interpretation of ECG exercise testing in adults. We discussed technical requirements and necessary equipment for the exercise testing laboratory as well as healthcare personnel competencies necessary to supervise ECG exercise testing and fully interpret test findings. Broad indications for ECG exercise testing include diagnostic assessment of coronary artery disease (CAD), including pre-test probability of $\mathrm{CAD}$, evaluation of functional disease severity and risk stratification in patients with established CAD, assessment of response to treatment, evaluation of exercise-related symptoms and exercise capacity, patient evaluation before exercise training/cardiac rehabilitation, and risk stratification prior to non-cardiac surgery. ECG exercise testing is safe if indications and contraindications are observed, testing is appropriately monitored, and indications for test termination are clearly established. The exercise protocol should be adjusted to the expected exercise capacity of a patient so as to limit the duration of exercise to 8-12 min. Clinical, haemodynamic, and ECG response to exercise is evaluated during the test. The test report should include information about the exercise protocol used, reason for test termination, perceived exertion, presence/severity of anginal symptoms, peak exercise capacity or tolerated workload in relation to the predicted exercise capacity, heart rate response, and the presence or absence of ST-T changes. The test report should conclude with a summary including clinical and ECG assessment.
\end{abstract}

Key words: electrocardiography, exercise stress testing

Kardiol Pol 2019; 77, 3: 399-408 


\section{INTRODUCTION}

Electrocardiographic (ECG) exercise stress test has been a major diagnostic test in cardiology for several decades. Ongoing technological advances and development of new guidelines have called for a revised and updated approach to the technique and interpretation of the ECG exercise testing. The present document outlines an expert opinion of the Polish Cardiac Society Working Group on Cardiac Rehabilitation and Exercise Physiology regarding the performance and interpretation of ECG exercise testing in adults. The present expert opinion was designed to be concise and brief, and a detailed discussion of issues relevant to exercise testing can be found in our previous statement published in 2016 [1]. The present expert opinion was developed based on that publication, the European Society of Cardiology (ESC) guidelines [2], and statements and guidelines of the American cardiac societies (American College of Cardiology [ACC] and American Heart Association [AHA]) [3-6].

\section{TECHNICAL REQUIREMENTS FOR EXERCISE STRESS TESTS \\ Personnel}

The personnel of the exercise testing laboratory should consist of medically-trained individuals experienced in exercise testing. The minimum required personnel includes one physician and one additional staff member (nurse, technician, or physiotherapist). Testing should be supervised by a physician experienced in exercise testing. To achieve the required expertise, it is necessary to perform and interpret at least 50 exercise tests under supervision. To maintain competence, it is necessary to perform at least 25 exercise tests a year [6].

\section{Skills needed for the performance and interpretation of the electrocardiographic exercise testing [6]}

\footnotetext{
Skills needed to supervise an exercise tests:

- Knowledge of indications for exercise testing

- Knowledge of contraindications to and risks of testing

- Knowledge of various exercise protocols and indications for their use

- Knowledge of preparation for exercise testing (skin preparation lead placement)

- Knowledge of end points of exercise testing and indications to terminate an exercise test

- Knowledge of basic exercise physiology, including haemodynamic response to exercise

- Knowledge of cardiac arrhythmias and the ability to recognise and treat serious arrhythmias

- Knowledge of the ECG evidence of myocardial ischaemia

- Knowledge of cardiovascular drugs and their effect on exercise performance, haemodynamic parameters, and the ECG
}

- Knowledge of the effects of age and disease on haemodynamic and ECG responses to exercise

- Ability to recognise and treat complications of exercise testing

- Ability to perform cardiovascular resuscitation

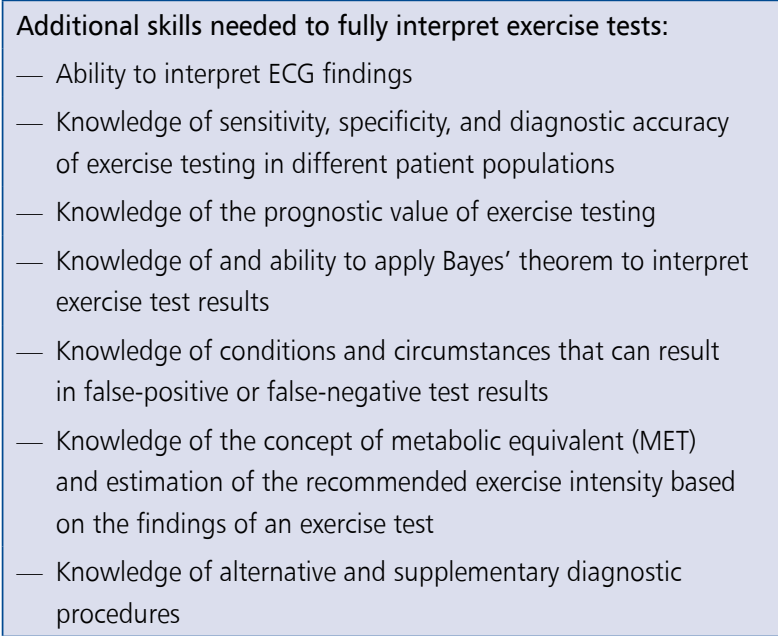

\section{Equipment for the exercise testing laboratory Testing room}

The exercise testing room should be large enough to allow an unobstructed access to the patient, including room for cardiopulmonary resuscitation and arrangement of patient transport in emergency situations, if necessary. The laboratory should be well lighted and well ventilated, with controlled temperature and humidity. The recommended room temperature is $20^{\circ} \mathrm{C}$ to $22^{\circ} \mathrm{C}$, with the recommended humidity of $50 \%$.

A scale of perceived exertion (6-20 or 0-10 Borg scale) (Table 1) and a 1-4 scale for the severity of angina and dyspnoea (Table 2) $[4,7,8]$ should be placed in clear view of the patient and personnel. The laboratory should be equipped with a readily available examination table, so the patient can be rested in a supine position after the testing if necessary.

The exercise testing laboratory should be equipped with an exercise testing system, device for blood pressure measurement, examination table, pulse oximeter (optionally), and equipment for cardiopulmonary resuscitation.

\section{Exercise testing system}

The exercise testing system, based on a treadmill or cycle ergometer, should be connected to a control module. The optimal solution is to have both treadmill and cycle ergometer available. A standard exercise testing laboratory does not have to be equipped with an arm ergometer.

It is recommended to use computer software for exercise testing that allows continuous monitoring of 12-lead ECG to evaluate ST-T changes, and it is acceptable to choose several leads to monitor cardiac rhythm. The system ought to allow data archiving. 
Table 1. Rating of perceived exertion - Borg scale and modified Borg scale

\begin{tabular}{|llll|}
\hline Borg scale & \multicolumn{2}{l|}{ Modified Borg scale } \\
\hline 6 & Very, very light & 0 & Nothing at all \\
8 & & 1 & Very, very weak \\
9 & Very light & 2 & Weak \\
10 & & 3 & Moderate \\
11 & Fairly light & 4 & Somewhat strong \\
12 & & 5 & Strong \\
13 & Somewhat hard & 6 & \\
14 & & 7 & Very strong \\
15 & Hard & 8 & \\
16 & & 9 & Very, very strong \\
17 & Very hard & & (almost maximum) \\
18 & & 10 & Maximum \\
19 & Very, very hard & & \\
20 & Maximum & & \\
\hline
\end{tabular}

Table 2. Severity scales of angina and dyspnoea

\begin{tabular}{|l|}
\hline Angina severity scale \\
$1+$ Onset of discomfort \\
$2+$ Moderate, bothersome \\
$3+$ Moderately severe \\
$4+$ Very severe \\
Dyspnoea severity scale \\
$1+$ Mild, noticeable to patient but not observer \\
$2+$ Mild, noticeable to observer \\
$3+$ Moderate difficulty but can continue \\
$4+$ Severe difficulty, patient cannot continue
\end{tabular}

Equipment for blood pressure measurement A device for blood pressure measurement should allow to select cuff size. Because of the interferences related to the functioning of the exercise testing system, devices for manual pressure measurement are preferred. It is recommended to choose an appropriate cuff size depending on the arm circumference. Cuffs should be disinfected after each use.

\section{Resuscitation trolley}

A resuscitation trolley should be located in a place that is clearly visible and easily accessible. The personnel should be trained in cardiopulmonary resuscitation, and a system of regular training and drills is recommended. A protocol for cardiopulmonary resuscitation should be clearly defined and established in accordance with the European Resuscitation Council guidelines $[9,10]$. A telephone with the number to be dialled to activate the resuscitation team (if such is available in a given institution) should be located in the laboratory or nearby. The laboratory should be equipped with a portable automated external defibrillator. Proper functioning of the device should be regularly checked by responsible staff members. The minimal set of medications should include syringes, intravenous infusion sets, infusion fluids $(0.9 \%$ saline, $5 \%$ dextrose), sublingual nitroglycerin, atropine, lidocaine, adenosine, adrenaline, amiodarone, dopamine, intravenous metoprolol, and acetylsalicylic acid (soluble 300 mg tablets).

\section{INDICATIONS AND CONTRAINDICATIONS TO ELECTROCARDIOGRAPHIC EXERCISE TESTING}

Indications and contraindications to electrocardiographic exercise testing should be always observed.

\section{Indications for electrocardiographic exercise testing}

Indications for electrocardiographic exercise testing $[1,2,3]$ :

- As the initial test for establishing a diagnosis of stable coronary artery disease (CAD) in patients with symptoms of angina and intermediate pre-test probability of CAD (15\%-65\%) (Table 3)

- Evaluation of the functional severity of $C A D$ (evaluation of coronary reserve)

- Risk stratification in patients with established CAD

- Evaluation of exercise capacity and exercise tolerance

- Patient evaluation prior to physical exercise/training

- Risk stratification prior to non-cardiac surgery (according to the ESC/ESA guidelines on non-cardiac surgery) [11]

- Evaluation of response to treatment (physical training, drug therapy, pacing)

- Assessment of exercise-induced symptoms (hypertensive response, chronotropic response, cardiac arrhythmias, symptoms related to valvular heart disease or cardiomyopathies)

\section{Contraindications to electrocardiographic exercise testing}
Absolute contraindications (according to AHA) [3]:
- Acute myocardial infarction (first two days)
- Unstable angina
- Uncontrolled cardiac arrhythmia with significant symptoms or haemodynamic compromise
- Active infective endocarditis
- Symptomatic severe aortic stenosis
- Decompensated heart failure
- Acute pulmonary embolism, pulmonary infarction, or deep vein thrombosis
- Acute myocarditis or pericarditis
- Acute aortic dissection
- Physical disability that precludes safe testing 
Table 3. Pre-test probability of coronary artery disease in stable patients with chest pain according to the European Society of Cardiology

\begin{tabular}{|c|c|c|c|c|c|c|}
\hline \multirow[t]{2}{*}{ Age [years] } & \multicolumn{2}{|c|}{ Typical angina } & \multicolumn{2}{|c|}{ Atypical angina } & \multicolumn{2}{|c|}{ Nonanginal pain } \\
\hline & Men & Women & Men & Women & Men & Women \\
\hline $30-39$ & 59 & 28 & 29 & 10 & 18 & 5 \\
\hline $40-49$ & 69 & 37 & 38 & 14 & 25 & 8 \\
\hline $50-59$ & 77 & 47 & 49 & 20 & 34 & 12 \\
\hline $60-69$ & 84 & 58 & 59 & 28 & 44 & 17 \\
\hline 70-79 & 89 & 68 & 69 & 37 & 54 & 24 \\
\hline$>80$ & 93 & 76 & 78 & 47 & 65 & 32 \\
\hline
\end{tabular}

The table shows estimated probability (\%) of the presence of a significant coronary artery obstruction.

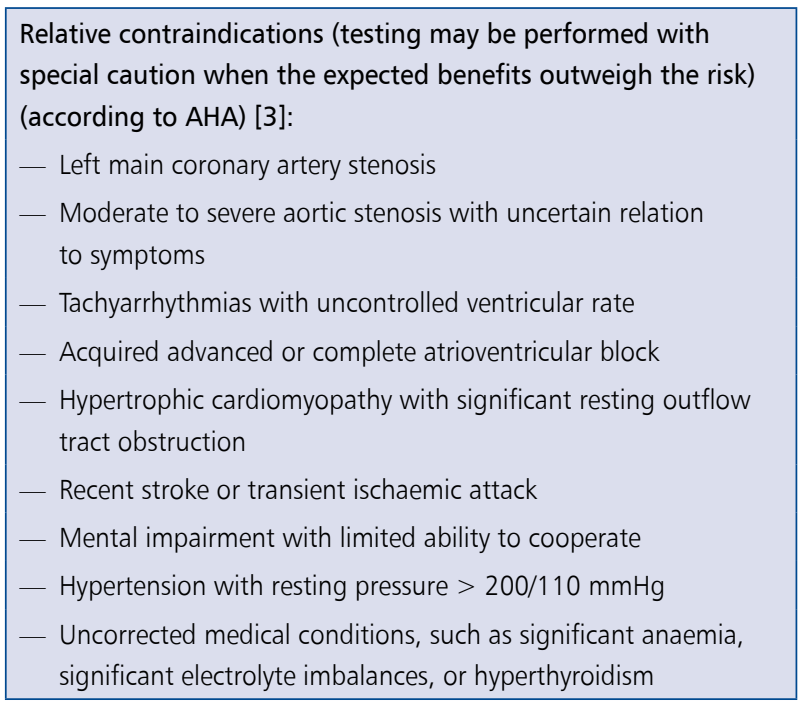

ECG changes that are a contraindication to the evaluation of CAD with ECG exercise testing $[2,5]$ :

- Persistent left bundle branch block

- Preexcitation syndromes

- Ventricular pacing

- Baseline ST depression $\geq 0.1 \mathrm{mV}$

— Digitalis-induced ST changes

\section{PREPARATION FOR TESTING Purpose of the test}

The purpose of the test should be indicated in the referral form or defined prior to the initiation of testing and noted in the test documentation.

\section{Patient medical history}

Before the testing, a short history should be taken regarding patient's major complaints and physical capacity, with particular attention to symptoms within the last week. Daily physical activity of the patient should be documented. The test documentation should include names and doses of cardiovascular medications taken by the patient, including the time when the last drug dose was administered on the day of the testing. Conditions of potential importance for the conduct and result of the testing (e.g. orthopaedic and neurological disorders, anginal symptoms, symptoms of heart failure, mental disorders such as depression or anxiety) should be documented.

\section{Patient data}

Before the test, a consecutive test number should be assigned and patient data should be entered to the system, including first name, family name, sex, date of birth, height, weight, patient contact data (address, telephone number), and the name of the referring physician.

\section{Stress testing protocol}

The test protocol should be selected according to the patient characteristics. Protocols with more intensive workload increments should be selected for young and fit patients, and protocols with more modest increases in workload between stages should be selected for older patients with reduced exercise capacity and concomitant conditions. The stress protocol should be chosen in such a way that a maximum/target exertion is reached between 8 and $12 \mathrm{~min}$ of the exercise stage.

\section{Maximum heart rate and chronotropic response}

The recommended formula for maximum heart rate is: 220 - age (in years) [12]. The maximum heart rate is used to calculate the target heart rate that is diagnostic for the diagnosis of CAD and is set at $85 \%$ of the maximum heart rate.

The chronotropic response is evaluated based on the percentage of the maximum heart rate at peak exercise, heart rate reserve, and the chronotropic index.

Heart rate reserve and the chronotropic index are calculated
using the following formulas:
- Heart rate reserve $=$ maximum heart rate - resting heart rate
- Chronotropic index $=[$ [peak heart rate during exercise - resting
heart rate) $/$ heart rate reserve $] \times 100 \%$


Table 4. Predicted exercise capacity in relation to age in men and women (in metabolic equivalent [MET])

\begin{tabular}{|c|c|c|c|c|c|}
\hline Age & Men* & Women** & Age & Men* & Women** \\
\hline 20 & 15.0 & 12.1 & 51 & 10.4 & 8.1 \\
\hline 21 & 14.9 & 12.0 & 52 & 10.2 & 7.9 \\
\hline 22 & 14.7 & 11.8 & 53 & 10.1 & 7.8 \\
\hline 23 & 14.6 & 11.7 & 54 & 9.9 & 7.7 \\
\hline 24 & 14.4 & 11.6 & 55 & 9.8 & 7.6 \\
\hline 25 & 14.3 & 11.5 & 56 & 9.6 & 7.4 \\
\hline 26 & 14.1 & 11.3 & 57 & 9.5 & 7.3 \\
\hline 27 & 14.0 & 11.2 & 58 & 9.3 & 7.2 \\
\hline 28 & 13.8 & 11.1 & 59 & 9.2 & 7.0 \\
\hline 29 & 13.7 & 10.9 & 60 & 9.0 & 6.9 \\
\hline 30 & 13.5 & 10.8 & 61 & 8.9 & 6.8 \\
\hline 31 & 13.4 & 10.7 & 62 & 8.7 & 6.6 \\
\hline 32 & 13.2 & 10.5 & 63 & 8.6 & 6.5 \\
\hline 33 & 13.1 & 10.4 & 64 & 8.4 & 6.4 \\
\hline 34 & 12.9 & 10.3 & 65 & 8.3 & 6.3 \\
\hline 35 & 12.8 & 10.2 & 66 & 8.1 & 6.1 \\
\hline 36 & 12.6 & 10.0 & 67 & 8.0 & 6.0 \\
\hline 37 & 12.5 & 9.9 & 68 & 7.8 & 5.9 \\
\hline 38 & 12.3 & 9.8 & 69 & 7.7 & 5.7 \\
\hline 39 & 12.2 & 9.6 & 70 & 7.5 & 5.6 \\
\hline 40 & 12.0 & 9.5 & 71 & 7.4 & 5.5 \\
\hline 41 & 11.9 & 9.4 & 72 & 7.2 & 5.3 \\
\hline 42 & 11.7 & 9.2 & 73 & 7.1 & 5.2 \\
\hline 43 & 11.6 & 9.1 & 74 & 6.9 & 5.1 \\
\hline 44 & 11.4 & 9.0 & 75 & 6.8 & 5.0 \\
\hline 45 & 11.3 & 8.9 & 76 & 6.6 & 4.8 \\
\hline 46 & 11.1 & 8.7 & 77 & 6.5 & 4.7 \\
\hline 47 & 11.0 & 8.6 & 78 & 6.3 & 4.6 \\
\hline 48 & 10.8 & 8.5 & 79 & 6.2 & 4.4 \\
\hline 49 & 10.7 & 8.3 & 80 & 6.0 & 4.3 \\
\hline 50 & 10.5 & 8.2 & & & \\
\hline
\end{tabular}

*Values for men calculated using the formula: MET $=18-(0.15 \times$ age $),{ }^{* *}$ Values for women calculated using the formula: MET $=14.7-(0.13 \times$ age $)$

- prior to the testing, it is important to know the type of the pacemaker, pacing mode, and rate-response function settings;

- heart rate response depends on the chronotropic competence and the mode of pacing;

- with fixed pacing rate, the testing should be guided by patient exertion and percentage predicted workload;

- exercise in a patient with an implanted cardioverter-defibrillator should be limited so as to increase the heart rate to 10-15 bpm below the arrhythmia detection threshold (optionally, the arrhythmia detection threshold may be temporarily set above the maximum heart rate, or the antiarrhythmic therapy functions may be disabled for testing);
- evaluation of ischaemia is not feasible in patients with ventricular pacing;

- loss of cardiac resynchronisation pacing during the testing is an indication to terminate the test.

\section{PATIENT PREPARATION FOR THE TESTING Informing the patient about the test and obtaining written consent}

Before the testing, the patient should be informed about the purpose and conduct of the test. Information about the test may be provided in writing or verbally. It is necessary to obtain a written consent for the test [1]. A sample consent form is shown in Table 5. 
Table 5. Exercise testing consent form

NAME OF THE LABORATORY

\section{ELECTROCARDIOGRAPHIC EXERCISE TESTING INFORMED CONSENT FORM}

PATIENT NAME AND SURNAME:

PERSONAL IDENTIFICATION (PESEL) NUMBER

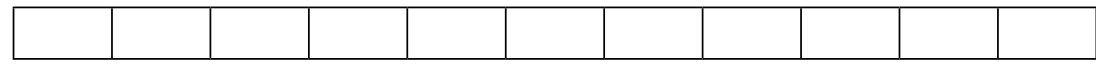

\section{Purpose of the testing}

Electrocardiographic exercise testing is performed to evaluate exercise capacity, diagnose coronary artery disease, evaluate coronary reserve, or assess response to treatment.

\section{Description of the testing}

The testing will be performed on a treadmill or a cycle ergometer under physician supervision. Single-use leads will be placed on the torso and connected to a computer that analyses the electrocardiogram. You will be asked to walk or cycle at a given rate, with the workload adjusted to your capabilities. The workload will be gradually increased by rising the treadmill speed and slope (or increasing pedal resistance of the cycle ergometer). When the desired parameters are reached, the physician will terminate the test. The test may also be terminated at any time at your request or if worrisome symptoms develop. During the test, the electrocardiogram will be monitored continuously, and blood pressure will be measured periodically, every 2-3 minutes. Exercise will be followed by several minutes of recovery, during which the electrocardiogram will be monitored and blood pressure will be measured.

\section{Information that should be disclosed to the healthcare personnel carrying out the test}

Any new symptoms that developed during the last few days (common cold, fever, chest pain etc.) should be reported before the test. Close cooperation with the healthcare personnel carrying out the test is necessary to achieve a meaningful test result. During the test, the patient should immediately report any complaints (pain, shortness of breath, dizziness, tinnitus etc.). However, it should be remembered that some degree of fatigue is normal during exercise testing and is not a reason for terminating the test. Premature termination of the test precludes obtaining the information expected by the referring physician.

\section{Possible complications/risks}

Exercise testing is a safe investigation but very rare cases of myocardial infarction and death have been reported. The most common complications include prolonged chest pain, arrhythmia, dizziness, fall in blood pressure, and leg pain. Some people may develop contact skin allergy in the areas of lead attachment. Fatigue may last up to several hours after the testing.

\section{PATIENT STATEMENT}

- I have got acquainted with the description of the proposed testing, its purpose, and possible risks that may arise due to the testing.

- I hereby declare that I have obtained satisfactory answers to my questions and I have fully understood the presented information.

\section{HEREBY GIVE AN INFORMED AND VOLUNTARY CONSENT TO THE PROPOSED TESTING}

\section{DO NOT GIVE CONSENT TO THE PROPOSED TESTING}

I have been informed about possible negative consequences of this decision for my health and life.

Physician taking the consent

\section{Patient preparation}

The patient should come in for the test about $3 \mathrm{~h}$ after a light meal, wearing comfortable, loose clothing and sport-type shoes that allow comfortable walking on the treadmill or cycling on the ergometer. The patient should avoid intensive exertion and refrain from drinking strong coffee, tea or energy drinks and smoking tobacco for $6 \mathrm{~h}$ before the testing. 
When exercise testing is performed for diagnostic purposes, it may be considered to withhold symptom-masking medications (e.g., $\beta$-blockers, nitrates) before the test. It is not recommended to withhold usual medications if the testing is performed to evaluate treatment response or exercise capacity.

\section{Skin preparation and lead placement}

Careful skin preparation before the testing is of key importance for the quality of the obtained ECG signal. A special paste should be used to remove superficial oils and the outermost epidermal layer. Excessive body hair in the areas of electrode application should be first shaved off. Adequate skin preparation should reduce skin resistance to $5000 \mathrm{ohm}$ or less. The Mason-Likar lead system should be used for lead placement on the torso [19].

\section{CONDUCT OF THE EXERCISE STRESS TESTING Exercise testing stages}

The test protocol should include a warm-up stage ( $2-3 \mathrm{~min}$ ), the exercise stage with graded (every $2-3 \mathrm{~min}$ ) or continuous increase in workload (optimal overall duration of 8-12 min), and a recovery period ( $5 \mathrm{~min}$ or until resolution of the ECG changes and/or clinical symptoms).

\section{Test protocols}

Graded or ramp protocols are used for testing at an increasing workload. Ramp protocols are recommended because of continuous increase in workload that shows a linear correlation with the haemodynamic and ECG response, and because of a more precise estimation of the exercise capacity. In addition, ramp protocols allow workload individualisation in patients with varying exercise capacity to achieve peak exertion within the optimal time frame during the testing. The proposed testing protocols are shown below.

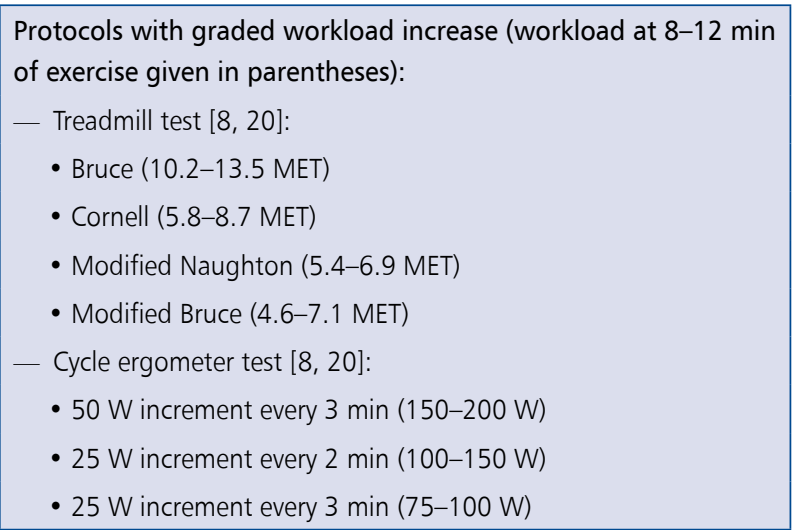

Protocols with continuous workload increase (ramp):

— Treadmill testing (workload at 8-12 min of exercise given in parentheses):

- BSU/Bruce ramp protocol according to Kaminsky et al. (9.4-13.9 MET) [21]
- American College of Sports Medicine (ACSM) ramp protocol (7.4-10.7 MET) [22]

- American Association of Cardiovascular and Pulmonary Rehabilitation (AACVPR) ramp protocol (6.5-10.1 MET) [23]

- Cycle ergometer testing - suggested ramp protocols for cycle ergometer testing according to the American Thoracic Society/ /American College of Chest Physicians (ATS/ACCP) (workload at $10 \mathrm{~min}$ of exercise) [24]:

- Ramp 30/min (300 W)

- Ramp 25/min (250 W)

- Ramp 20/min (200 W)

- Ramp 15/min (150 W)

- Ramp 10/min (100 W)

- Ramp 5/min (50 W)

\section{INDICATIONS FOR TERMINATION OF EXERCISE TESTING}

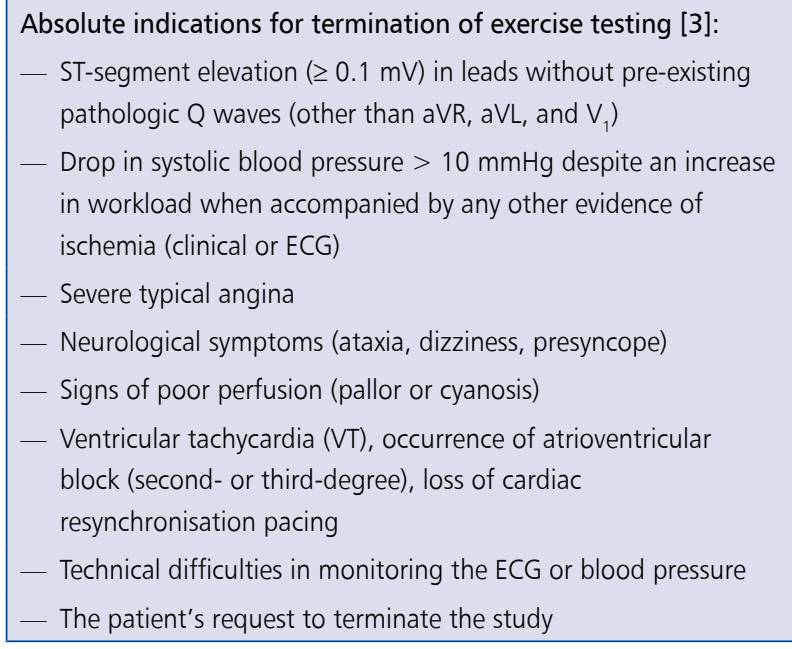

Relative indications for termination of exercise testing [3]:

- Rapidly progressing ST depression (horizontal or downsloping) ( $>2 \mathrm{~mm}$ ) or a sudden change in the cardiac electrical axis

- Drop in systolic blood pressure $>10 \mathrm{mmHg}$ despite an increase in workload in the absence of other evidence of ischaemia

— Increasing chest pain

- Severe fatigue, wheezing, leg cramps, claudication

- Arrhythmias other than VT, i.e., multifocal single ventricular ectopy, ventricular couplets, ventricular bigeminy, supraventricular tachycardia, bradyarrhythmias

- Exaggerated hypertensive response (systolic blood pressure $>250 \mathrm{mmHg}$ or diastolic blood pressure $>115 \mathrm{mmHg}$ )

- Development of bundle branch block or intraventricular conduction disturbances that cannot be distinguished from VT

\section{PARAMETERS EVALUATED DURING EXERCISE TESTING}

Clinical, haemodynamic, and ECG parameters should be evaluated during exercise testing $[1,3,4]$. 
Clinical parameters:

- Anginal pain (severity, increase, timing)

- Degree of perceived exertion (rated using Borg scale)

- Peak exercise capacity (MET) for treadmill testing or peak tolerated workload (W) for cycle ergometer testing

- Relative exercise capacity (\%)

- Duration of exercise (minutes and seconds)

- Occurrence of symptoms necessitating test termination (dizziness, fatigue, shortness of breath, leg pain)

\section{Haemodynamic parameters:}

- Systolic and diastolic blood pressure at each workload stage

- Heart rate at rest, peak workload and 1 min of recovery

- Achieved percentage of maximum heart rate (\%)

- Heart rate decrease at $1 \mathrm{~min}$ of recovery

- Maximum heart rate for a given patient

- Chronotropic response (achieved percentage of maximum heart rate, heart rate reserve, chronotropic index)

- Double product (peak systolic blood pressure multiplied by peak heart rate)

- Dynamics of systolic and diastolic blood pressure changes

\section{ECG parameters:}

- 12-lead ECG should be recorded from the torso leads at baseline, at peak workload, and during recovery

- Assessment of ST changes in at least three consecutive cycles, taking into account averaging of computer-generated signal and raw data (to exclude artefacts) at baseline, during each workload stage, and during the recovery stage for at least 5 min or until resolution of ST changes and return of heart rate and blood pressure to baseline values

- Baseline ST depression should be taken into account

- Measurement of ST changes at 60-80 ms after the J point

- Assessment of the type of ST depression - horizontal, downsloping, upsloping

- Number of leads with ST changes, timing of their recovery, and the characteristics of changes during the recovery phase

- Assessment of arrhythmias and conduction disturbances

\section{TEST REPORT AND CONCLUSIONS}

The test report should include information about $[1,3,4,18]$ :

- Test protocol

- Reason for test termination (fatigue, pain, ECG changes, arrhythmia, heart rate limit, other)

- Perceived exertion (rated using Borg scale)

- Severity of pain

- Peak exercise capacity (MET) or peak tolerated workload (W) (for cycle ergometer testing)

- Relative exercise capacity (\%)
- Heart rate at peak exercise and achieved percentage of maximum heart rate

- Maximum blood pressure values

- Description of exercise-induced ST changes or their absence (characteristics, severity, number of leads, timing of recovery)

- ST/heart rate index ( $\mathrm{mV} / \mathrm{bpm}$ ) (recommended optionally, if calculation is technically feasible)

- Heart rate decrease at 1 min of recovery

- Chronotropic response taking into account drugs taken by the patient

- Description of arrhythmias and conduction disturbances if present (in particular ventricular arrhythmia during recovery)

- Duke treadmill score - calculator available at: http://www.cardiology.org/tools/medcalc/duke/

- It is recommended to report the test result as "positive" or "negative" (clinically or electrocardiographically) in the case of testing performed for the diagnosis of CAD, while in other cases, the terms "normal" or "abnormal" are preferred, with description of the identified abnormalities

- In the case of testing performed for the diagnosis of CAD, the term "nondiagnostic test" should be used if $85 \%$ of the maximum age-predicted heart rate has not been achieved

- Final conclusion based on clinical and ECG evaluation, with possible suggestions regarding further investigations

\section{TEST RESULT ARCHIVING}

The test report is handed to the patient in a paper form, authorised by the physician who supervised and interpreted the test, and it should include at least a title page with patient data and test result interpretation, a summary of exercise parameters, and pages with averaged lead tracings from each testing stage with ST changes shown. It is recommended to archive results in an electronic form in at least two copies (in case of data loss) - in the memory of the computer used for the exercise testing and a backup copy (using external memory or a data server).

\section{DURATION OF THE TEST, FUNDING, AND REIMBURSEMENT}

Electrocardiographic exercise testing is a separate procedure. It should be separately billed, taking into account the cost of labour of the nurse/technician and the physician supervising and interpreting the test. The minimum time for patient preparation and test conduct is $30 \mathrm{~min}$, but it may be extended to $60 \mathrm{~min}$ in case of complications necessitating prolonged patient follow-up or implementation of appropriate measures (e.g., when urgent hospitalisation is needed).

\section{SUMMARY}

Electrocardiographic exercise testing remains a major diagnostic and investigative tool in contemporary cardiology practice. Although its diagnostic importance has somewhat decreased 
in the current era of rapid development of anatomical and functional imaging methods, its prognostic role is increasing not only in CAD but also in other cardiovascular conditions.

\section{Conflict of interest: none declared}

\section{References}

1. Straburzyńska-Migaj E, Bednarz B, Piotrowicz R, et al. Testy wysiłkowe. Stanowisko Ekspertów Sekcji Rehabilitacji Kardiologicznej i Fizjologii Wysiłku, Sekcji Elektrokardiologii Nieinwazyjnej i Telemedycyny, Sekcji Kardiologii Sportowej Polskiego Towarzystwa Kardiologicznego. Via Medica, Gdańsk. 2016.

2. Montalescot G, Sechtem U, Achenbach S, et al. 2013 ESC guidelines on the management of stable coronary artery disease: the Task Force on the management of stable coronary artery disease of the European Society of Cardiology. Eur Heart J. 2013; 34(38): 2949-3003, doi: 10.1093/eurheartj/eht296, indexed in Pubmed: 23996286.

3. Fletcher GF, Ades PA, Kligfield P, et al. Exercise standards for testing and training: a scientific statement from the American Heart Association. Circulation. 2013; 128(8): 873-934, doi: 10.1161/CIR.0b013e31829b5b44, indexed in Pubmed: 23877260.

4. Myers J, Arena R, Franklin B, et al. Recommendations for clinical exercise laboratories: a scientific statement from the american heart association. Circulation. 2009; 119(24): 3144-3161, doi: 10.1161/CIRCULATIONAHA.109.192520, indexed in Pubmed: 19487589.

5. Gibbons RJ, Balady GJ, Bricker JT, et al. A report of the American College of Cardiology/American Heart Association Task Force on Practice Guidelines (Committee to Update the 1997 Exercise Testing Guidelines). J Am Coll Cardiol. 2002; 40(8): 1531-1540.

6. Rodgers GP, Ayanian JZ, Balady G, et al. American College of Cardiology/American Heart Association Clinical Competence statement on stress testing: a report of the American College of Cardiology/American Heart Association/American College of Physicians - American Society of Internal Medicine Task Force on Clinical Competence. J Am Coll Cardiol. 2000; 36(4): 1441-1453, indexed in Pubmed: 11028516.

7. Borg GA. Psychophysical bases of perceived exertion. Med Sci Sports Exerc. 1982; 14(5): 377-381, indexed in Pubmed: 7154893.

8. Froelicher VF: „Metody”. In: Froelisher VF. Podręcznik testów wysiłkowych. Bel Corp Warszawa. 1999.

9. Koster RW, Baubin MA, Bossaert LL, et al. European Resuscitation Council Guidelines for Resuscitation 2010 Section 2. Adult basic life support and use of automated external defibrillators. Resuscitation. 2010; 81(10): 1277-1292, doi: 10.1016/j.resuscitation.2010.08.009, indexed in Pubmed: 20956051.

10. Deakin CD, Nolan JP, Soar J, et al. European Resuscitation Council Guidelines for Resuscitation 2010 Section 4. Adult advanced life support. Resuscitation. 2010; 81(10): 1305-1352, doi: 10.1016/j. resuscitation.2010.08.017, indexed in Pubmed: 20956049.

11. Kristensen SD, Knuuti J, Saraste A, et al. 2014 ESC/ESA Guidelines on non-cardiac surgery: cardiovascular assessment and management: The Joint Task Force on non-cardiac surgery: cardiovascular assessment and management of the European Society of Cardiology (ESC) and the European Society of Anaesthesiology (ESA). Eur Heart J. 2014; 35(35): 2383-2431, doi: 10.1093/eurheartj/ehu282, indexed in Pubmed: 25086026.

12. Astrand I. Aerobic work capacity in men and women with special reference to age. Acta Physiol Scand Suppl. 1960; 49(169): 1-92, indexed in Pubmed: 13794892.

13. Morris CK, Myers J, Froelicher VF, et al. Nomogram based on metabolic equivalents and age for assessing aerobic exercise capacity in men. J Am Coll Cardiol. 1993; 22(1): 175-182, indexed in Pubmed: 8509539.

14. Gulati M, Black HR, Shaw LJ, et al. The prognostic value of a nomogram for exercise capacity in women. N Engl J Med. 2005; 353(5): 468-475, doi: 10.1056/NEJMoa044154, indexed in Pubmed: 16079370.

15. Jones NL, Makrides L, Hitchcock C, et al. Normal standards for an incremental progressive cycle ergometer test. Am Rev Respir Dis. 1985; 131(5): 700-708, doi: 10.1164/arrd.1985.131.5.700, indexed in Pubmed: 3923878.

16. Myers J, Bader D, Madhavan R, et al. Validation of a specific activity questionnaire to estimate exercise tolerance in patients referred for exercise testing. Am Heart J. 2001; 142(6): 1041-1046, doi: 10.1067/mhj.2001.118740, indexed in Pubmed: 11717610 .

17. Jurca R, Jackson AS, LaMonte MJ, et al. Assessing cardiorespiratory fitness without performing exercise testing. Am J Prev Med. 2005; 29(3): 185-193, doi: 10.1016/j.amepre.2005.06.004, indexed in Pubmed: 16168867.

18. Kligfield P, Lauer MS. Exercise electrocardiogram testing: beyond the ST segment. Circulation. 2006; 114(19): 2070-2082, doi: 10.1161/CIRCULATIONAHA.105.561944, indexed in Pubmed: 17088475.

19. Mason RE, Likar I. A new system of multiple-lead exercise electrocardiography. Am Heart J. 1966; 71(2): 196-205, indexed in Pubmed: 5902099.

20. Rybicki J. Zasady doboru protokołu testu wysiłkowego u chorych i zdrowych nietrenujących wyczynowo. In: Dłużniewski M, Kalarus Z, Pikto-Pietkiewicz W, et al. Sport wyczynowy i rekreacyjny - problemy kardiologa i internisty. Wydanie II rozszerzone i uaktualnione. Czelej, Warszawa. 2017.

21. Kaminsky LA, Whaley MH. Evaluation of a new standardized ramp protocol: the BSU/Bruce Ramp protocol. J Cardiopulm Rehabil. 1998; 18(6): 438-444, indexed in Pubmed: 9857276.

22. American College of Sports Medicine. ACSM's Guidelines for exercise testing and prescription. 9th edition. Lippincott Williams \& Wilkins, Baltimore. 2014

23. American Association of Cardiovascular and Pulmonary Rehabilitation. Guidelines for Cardiac Rehabilitation and Secondary Prevention Programs. 4th Edition. Human Kinetics, Champaign. 2004.

24. American Thoracic Society, American College of Chest Physicians. ATS/ACCP Statement on cardiopulmonary exercise testing. Am J Respir Crit Care Med. 2003; 167(2): 211-277, doi: 10.1164/rccm.167.2.211, indexed in Pubmed: 12524257.

Cite this article as: Smarż K, Jaxa-Chamiec T, Bednarczyk T, et al. Electrocardiographic exercise testing in adults: performance and interpretation. An expert opinion of the Polish Cardiac Society Working Group on Cardiac Rehabilitation and Exercise Physiology. Kardiol Pol. 2019; 77(3): 399-408; doi: 10.5603/KP.a2018.0241. 